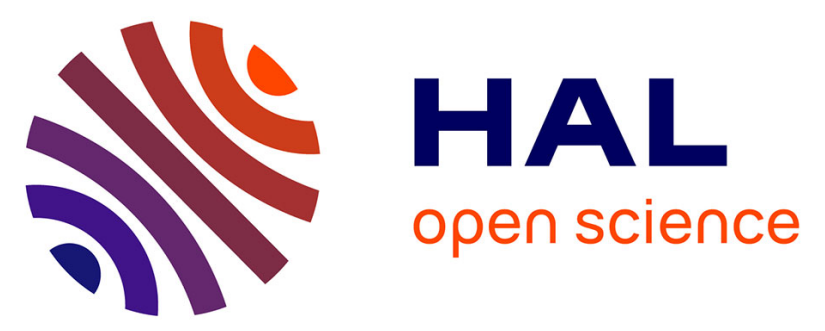

\title{
Access of Asymmetric Fluid Velocity Profile in High Precision Ultrasonic Velocimetry
}

Emmanuelle Mandard, Denis Kouamé, Rodolphe Battault, Jean-Pierre

Remenieras, Frédéric Patat

\section{- To cite this version:}

Emmanuelle Mandard, Denis Kouamé, Rodolphe Battault, Jean-Pierre Remenieras, Frédéric Patat. Access of Asymmetric Fluid Velocity Profile in High Precision Ultrasonic Velocimetry. IEEE International Ultrasonics Symposium (2006), IEEE- UFFC Society, Oct 2006, Vancouver, British Columbia, Canada. 10.1109/ULTSYM.2006.262 . hal-03149782

\section{HAL Id: hal-03149782 \\ https://hal.science/hal-03149782}

Submitted on 25 Feb 2021

HAL is a multi-disciplinary open access archive for the deposit and dissemination of scientific research documents, whether they are published or not. The documents may come from teaching and research institutions in France or abroad, or from public or private research centers.
L'archive ouverte pluridisciplinaire HAL, est destinée au dépôt et à la diffusion de documents scientifiques de niveau recherche, publiés ou non, émanant des établissements d'enseignement et de recherche français ou étrangers, des laboratoires publics ou privés. 


\title{
Access of asymmetric fluid velocity profile in high precision ultrasonic velocimetry
}

\author{
E. Mandard * , D. Kouamé *, R. Battault ${ }^{\dagger}$, J.-P. Remenieras * and F. Patat * \\ *Université François Rabelais - LUSSI/ CNRS FRE 2448, 10 bd Tonnellé BP2332 37032 Tours Cedex, France \\ Email: denis.kouame@univ-tours.fr \\ $\dagger$ Faure Herman, Route de Bonnétable, BP 2015472406 La Ferté Bernard, France \\ Email: calzina@faureherman.com
}

\begin{abstract}
A transit time ultrasonic flowmeter is a very accurate tool to measure the volumetric flowrate of a fluid flowing in a pipe. To estimate the flowrate, the fluid velocity averaged over the cross-section of the pipe has to be estimated from the fluid velocities averaged over the paths of the flowmeter. That is the reason why the velocity profile of the fluid, which depends on not only the Reynolds number but also on the upstream and downstream pipe configuration, is of great interest in transit time flowmetering. In this work, we propose to reconstruct the fluid velocity profile by using the measures of a 18 paths flowmeter measures to offer an in situ diagnostic tool of the flow. We first test tomography methods: filtered back-projection and then an iterative one, the Algebraic Reconstruction Technique (ART). These methods are not really appropriate to our context of incomplete data. We have previously [1] defined a theoretical parametric model of the velocity profile which allows to in situ detect and characterize the asymmetry of the flow. We defined in this work a new parametric model which has a flowrate function of the asymmetry of the profile. It also has the advantage of determining the parameters of the symmetric term of the model in taking into account the asymmetric part of the profile. This new profile is a promising way in increasing the accuracy of the flowmeter in disturbed flow condition by taking into account the additional information provided by the velocity profile reconstruction.
\end{abstract}

\section{INTRODUCTION}

A transit time ultrasonic flowmeter is an accurate tool to measure the volumetric flow rate of a fluid flowing in a pipe. Transit time method is based on a well known principle. Let $A$ and $B$ two transducers located on each side of a pipe like presented on Fig. 1. Transit time $T_{A \rightarrow B}$ of the downstream

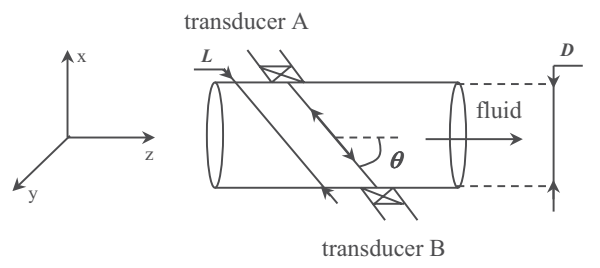

Fig. 1. Transit time principle.

signal and $T_{B \rightarrow A}$ of the upstream signal can be expressed as,

$$
T_{A \rightarrow B}=\int_{0}^{L} \frac{d l}{c+v_{z}(l) \cos \phi}
$$

and

$$
T_{B \rightarrow A}=\int_{0}^{L} \frac{d l}{c-v_{z}(l) \cos \phi}
$$

where $L$ is the path length, $c$ the velocity of sound, $v_{z}(l)$ the axial flow velocity measured at point $l$ along the path and $\phi$ the inclination angle. The expression of the path velocity can be obtained by substituting (1) into (2),

$$
v_{\text {path }}=\frac{L\left(T_{B \rightarrow A}-T_{A \rightarrow B}\right)}{2 T_{A \rightarrow B} T_{B \rightarrow A} \cos \phi} .
$$

The transit time method allows to measure the fluid velocity averaged over the path between a pair of transducers. The most accurate flowmeters measure several path velocities. To estimate the volumetric flow rate, the fluid velocity averaged over the cross-section of the pipe has to be estimated from these path velocities. The velocity of the fluid is not uniform along the pipe cross-section. In fact, the velocity profile depends on the Reynolds number of the flow and on the upstream and downstream pipe geometry [2]. So, the fluid velocity profile is of great interest in the context of transit time flowmetering. That is the reason why we propose to reconstruct this velocity profile thanks to the path velocities measured by a transit time flowmeter. It will offer an in situ diagnostic tool of the flow.

The flowmeter used during this work owns 18 paths generated by 12 three-directional transducers. Transducers are arranged on two rings at a distance $D$ where $D$ is the diameter of the pipe. When the 18 paths are projected on the crosssection of the pipe, they appeared as 9 chords like presented on Fig. 2. On this figure, each little circle represent two transducers: one of the upstream ring et one of the downstream ring (used in apostrophe). We can see that a chord on Fig. 2 comes from two paths. These paths, which own the same projection on the cross-section of the pipe are referred to as crossed paths. Considering the average of fluid velocities measured on crossed paths allows to entirely compensates for the swirl which is the whole of non-flowing transverse velocities of the fluid [1]. The 18 paths of the flow meter consist of 6 paths which pass by the center of the pipe and will be referred to as direct paths and 12 paths at a distance $\frac{R}{2}$ (where $R$ is the radius of the pipe) of the center of the pipe and will be referred to as indirect paths. 

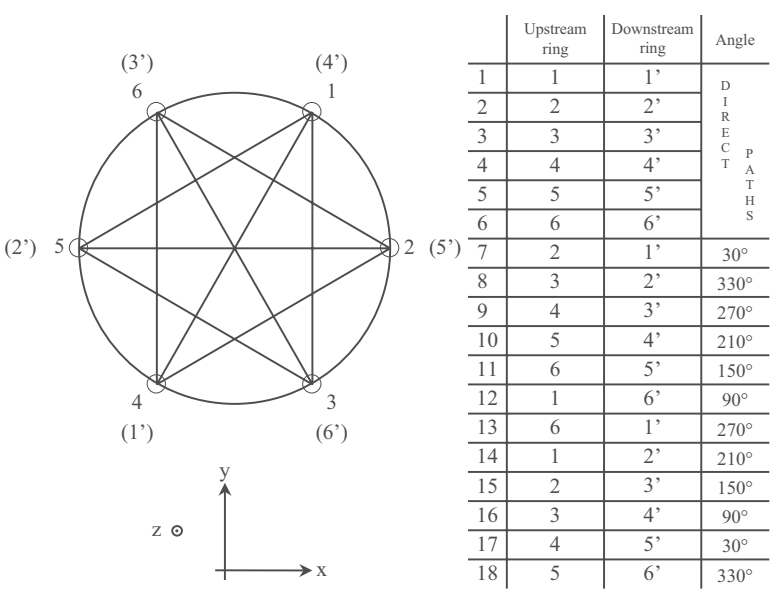

Fig. 2. 2D paths configuration of flowmeter used during this work.

To reconstruct the fluid velocity profile from the velocities measured by this flowmeter, we first investigated about tomography methods. We first investigated the filtered backprojection and then an iterative method: the Algebraic Reconstruction Method (ART). But in our context of incomplete data, these methods provided poor results. We have previously defined a theoretical parametric model of the fluid velocity profile and a method to estimate the parameters from the measures of the flowmeter [1]. This model allows to, in situ, detect and characterize asymmetry of the fluid velocity profile. Therefore, the flow rate of the forementioned theoretical model does not take into account the asymmetry of the profile. To overcome this limitation, we propose here a new profile which is more appropriate to a use of the information provided by the velocity profile reconstruction to improve the performances of existing ultrasonic flowmeter accuracy.

\section{TOMOGRAPHY METHODS}

The basic principle of tomography is that a subject is interrogated by a sensor from many different viewing angles in a cross-section and the measured data are used to reconstruct an image representing the material distribution. We can see on Fig. 2 that, if we considered that velocity measures are realized on the cross-section of the pipe and averaged on crossed paths, the configuration of the flowmeter paths allows to think about tomography reconstruction of the fluid velocity profile.

The most classical method of tomography probably is the filtered back-projection. The Radon transform $F(l, \theta)$ of the function $f(x, y)$ is the line integral of $f(x, y)$ on the line characterized by the distance $l$ and the angle $\theta$. The Projection Slice Theorem (PST) states that the Fourier transform of the Radon transform of $f(x, y)$ is equal to the 2D Fourier transform on a line at an angle $\theta$ of the function $f(x, y)$. So, if some points of the Radon transform of $f(x, y)$ are known, it is possible to reconstruct $f(x, y)$. The filtered back-projection consist in filtering the data before the back-projection to eliminate the star like artefacts.

The flowmeter provides us 9 points of the Radon transform of the fluid velocity profile. In this context of incomplete data, an interpolation of data is necessary. The result of the reconstruction of a theoretical velocity profile defined in [3] is presented on the Fig. 3. In our case, the reconstruction is poor. In fact, the filtered back-projection is currently used in the medical domain but in case of a great number of data. So, we then used a technique which is more appropriate to incomplete data : the Algebraic Reconstruction Technique (ART). The
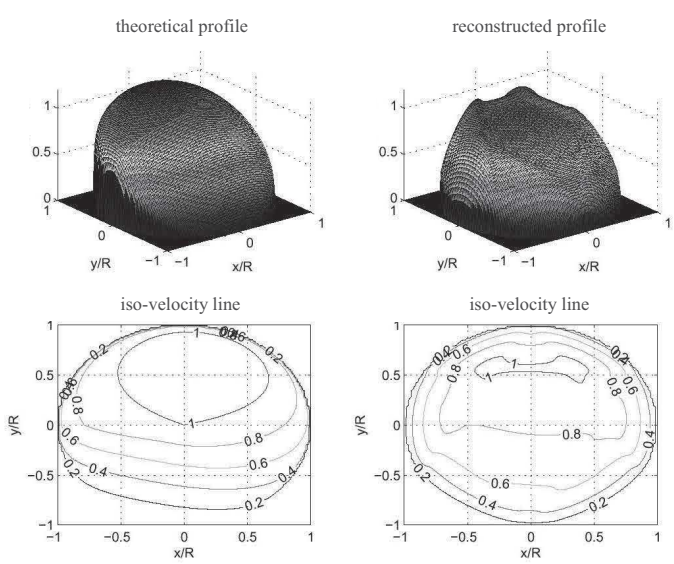

Fig. 3. Example of the reconstruction of a theoretical profile by filtered back-propagation.

Algebraic Reconstruction Technique is an iterative method introduced in [4]. It solves the reconstruction problem on a simple intuitive basis. Each projection is thrown back across the higher dimensional region whence it came, with repeated corrections to bring each projection of the estimate into agreement with the corresponding measured projection. The application of this algorithm needs the careful modelling of the taking of measure. The difficulty of the ART is the fact that it only updates the pixels of the profile which are taken into account in the measures. So, interpolation is required to transmit the information from the chords to the entire cross-section of the pipe. Fig. 4 presents the result of the reconstruction of a theoretical profile defined in [3] by the ART. Once again, the result is poor. Classical methods of

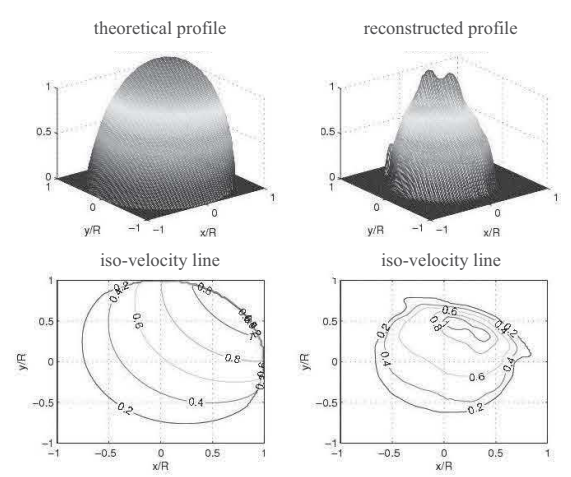

Fig. 4. Example of the reconstruction of a theoretical profile by ART. 


$$
V(r, \theta)=V_{0}\left(1-\frac{r}{R}\right)^{\frac{1}{n}}+\sin \left[\pi\left(1-\frac{r}{R}\right)\right]\left\{a_{1} \sin ^{2}\left(\frac{\theta}{2}-\theta_{1}\right)+a_{2} \sin ^{2}\left(\theta-\theta_{2}\right)\right\}
$$

tomography are not appropriate to our context of incomplete data. So, we decided to use a theoretical parametric model to reconstruct the fluid velocity profile by the use of the flowmeter.

\section{PARAMETRIC MODEL}

To reconstruct the fluid velocity profile from path velocities measured by the transit time flowmeter, we have defined a first theoretical parametric model [1]. This model allows to detect and characterize an asymmetry of the fluid velocity profile. Therefore, the flow rate of this profile does not take into account the asymmetry of the profile. So, to overcome this limitation, we defined an other profile defined in (4).

Like the first defined model in [1], a power law $V_{0}(1-$ $\left.\frac{r}{R}\right)^{\frac{1}{n}}$ is used to describe the symmetric part of this model. $V_{0}$ is the fluid velocity at the center of the pipe and $n$ a parameter function of the Reynolds number of the flow. The asymmetric part of the profile consists of two terms: a one-pole term (parameters $a_{1}$ et $\theta_{1}$ ) and a two-pole term (parameters $a_{2}$ et $\theta_{2}$ ). The one-pole term can generate an off-center maximum of the profile. $a_{1}$ and $\theta_{1}$ are respectively the strength and the direction of the one-pole asymmetry of the model. The twopole term can generate two off-center maxima of the profile. $a_{2}$ and $\theta_{2}$ are respectively the strength and the direction of the two-pole asymmetry of the model.

The parameters $a_{1}, \theta_{1}, a_{2}$ and $\theta_{2}$ of the asymmetric part of the model are determined from velocities measured on indirect chords i.e. chords which do not pass by the center of the pipe. In fact, according to our model, in case $a_{2}=0$, the curve representing the velocities measured on indirect chords in function of the angle of the chord (cf. Fig. 2) is a sine one presented one Fig. 5. The amplitude of this curve matches

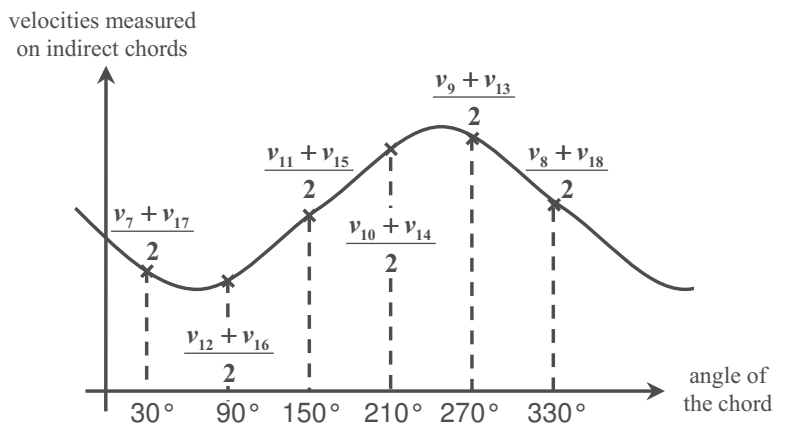

Fig. 5. Example of the reconstruction of a theoretical profile by ART.

the parameter $a_{1}$ which traduces the strength of the one-pole asymmetry. The phase displacement of the curve matches the parameter $\theta_{1}$ of the one-pole asymmetry. The parameters $a_{2}$ and $\theta_{2}$ are respectively linked with the amplitude and the phase displacement of the two-period sine curve which pass by the 6 aforementioned points. The parameters $a_{1}, a_{2}, \theta_{1}$ and $\theta_{2}$ are inferred from the Fourier coefficients of the indirect chord velocities.

The fluid velocities measured on direct paths which pass by the center of the pipe are higher than those measured on indirect paths. So, the ratio $\frac{V_{d}}{V_{i}}$ where $V_{d}$ is the mean of the fluid velocities measured on direct paths and $V_{i}$ the mean velocities measured on indirect paths is greater than 1 . So, the ratio $\frac{V_{d}}{V_{i}}$ matches the slope of the velocity profile: it is high during the laminar regime (low Reynolds) and decrease when the Reynolds number increase. The parameter $n$ of the Power law, function of the Reynolds, is inversely proportional to the ratio $\frac{V_{d}}{V_{i}}$. The relation between this ratio and the parameter $n$ was determined in simulation and is presented on Fig. 6.

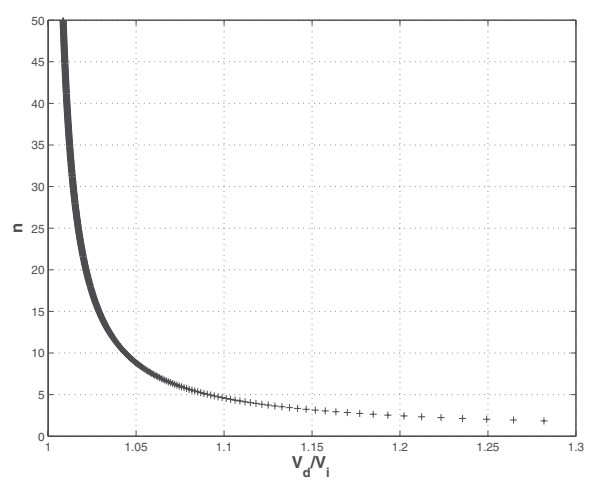

Fig. 6. Relation, determined in simulation, between the parameter $n$ of the power law and the ratio $\frac{V_{d}}{V_{i}}$.

To determine the parameter $n$ of the model, we have to take into account not only the ratio $\frac{V_{d}}{V_{i}}$ but also the asymmetric part of the theoretical model. Doing so,

$$
n=\frac{p_{1}}{\frac{V_{d}}{V_{c}}+q_{1}}
$$

where $p_{1}$ and $q_{1}$ are functions of the parameter $V_{0}$ and $a=$ $a_{1}+a 2 . p_{1}$ and $q_{1}$ were determined by simulation in function of $V_{0}$ and $a$. The parameter $V_{0}$ can be estimated like that:

$$
V_{0}=\frac{n+1.034}{n-0.2519}\left(\sum_{i} v_{i}-0.352 a\right)
$$

where $v_{i}$ is the path velocity measured on path $i$.

To determine the parameters $n$ and $V_{0}$, we first initialize them and then, make some iterations with (5) an (6). $n$ can be initialized by considering that the profile is a Power law. $n$ is 
thus determined from the ratio $\frac{V_{d}}{V_{i}} c f$. Fig. 6. The flowrate of the power law

$$
Q=\int_{0}^{2 \pi} \int_{0}^{R} V_{0}\left(1-\frac{r}{R}\right)^{\frac{1}{n}} r d r d \theta=\frac{2 \pi V_{0} R^{2} n^{2}}{(n+1)(2 n+1)}
$$

allows to initialize the parameter $V_{0}$ from $n$ and the flow rate measured by the flowmeter. To give an example, we will reconstruct the theoretical profile presented on Fig. 7. This profile is coming from (4) with $V_{0}=1, n=10, a_{1}=0.1$, $\theta_{1}=0^{\circ}, a_{2}=0.2$ and $\theta_{2}=0^{\circ}$. The parameters of the

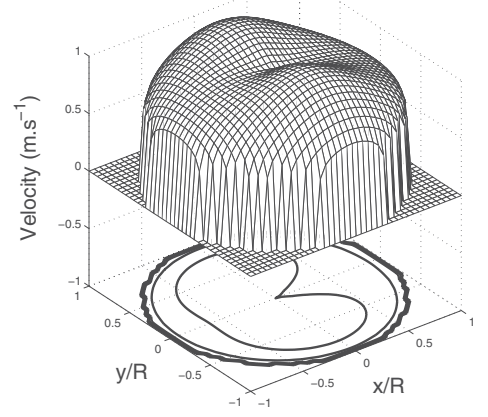

Fig. 7. Theoretical profile to be reconstructed.

asymmetric part are first estimated from the indirect chordal velocities :

$$
\begin{cases}\hat{a_{1}}=1.0003 \cdot 10^{-1} & \hat{\theta_{1}}=\left(10^{-13}\right)^{\circ} \\ \hat{a_{2}}=2.0001 \cdot 10^{-1} & \hat{\theta_{2}}=\left(10^{-14}\right)^{\circ}\end{cases}
$$

These estimated parameter of the asymmetrical part are very close to theoretical ones. By assuming that the profile is a Power law, we can initialize the parameters $V_{0}$ and $n$ :

$$
\left\{\begin{array}{l}
\hat{V}_{0 i n i}=1.041 \\
\hat{n}_{\text {ini }}=18.35
\end{array}\right.
$$

The initialization of the reconstructed profile is coming from (8) and (9) is presented on Fig. 8. We can note here that the asymmetry of the profile do not allow a good estimation of the Reynolds number by the ratio $\frac{V_{d}}{V_{i}}$. The estimation of $V_{0}$ is affected by the asymmetry to a lesser extent. Finally, the parameters become

$$
\left\{\begin{array}{l}
\hat{V}_{0}=1.0007 \\
\hat{n}=9.95
\end{array}\right.
$$

Four iterations are sufficient to a correct estimation of the parameters of the theoretical model. Unlike the first model which was defined, the parameters $n$ and $V_{0}$ of the term in Power law are estimated by taking into account the asymmetry of the profile.

\section{CONCLUSION}

To develop an in situ diagnostic tool of the flow, we reconstruct the fluid velocity profile thanks to the path velocities measured by a transit time ultrasonic flowmeter. We first test tomography methods: filtered back-projection and an iterative

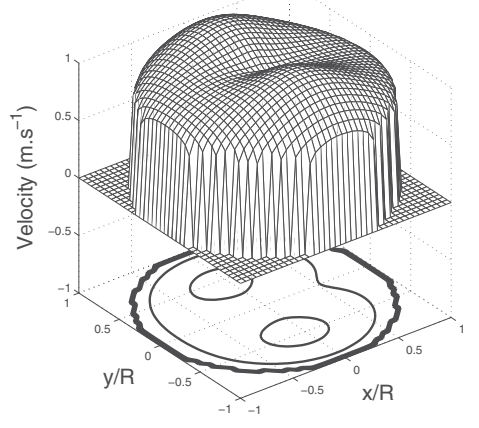

Fig. 8. Initialization of the reconstructed profile.

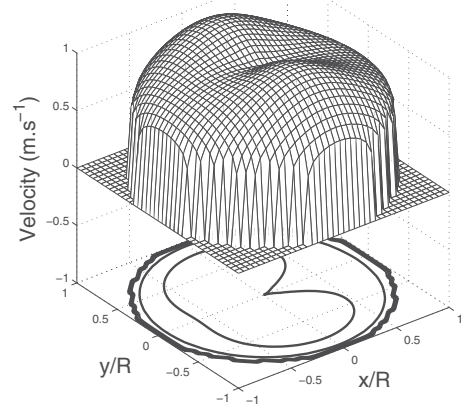

Fig. 9. Reconstructed profile.

one, the Algebraic Reconstruction Technique (ART). These methods are not actually appropriate to our context of incomplete data. We have previously defined a theoretical parametric model which allows to in situ detect and characterize the asymmetry of the flow profile [1]. The new model proposed here has the advantage of having a flowrate which is function not only of the parameters of the symmetric term of the parametric model but also of the parameters of the asymmetric terms. It has an additional advantage that is the fact that the parameters of the term in Power law $V_{0}$ and $n$ are determined as function of the asymmetry of the profile. This new model is a promising way to increase the accuracy of the flowmeter in disturbed flow conditions by taking into account the additional information provided by the velocity profile reconstruction.

\section{REFERENCES}

[1] E. Mandard, D. Kouamé, R. Battault, J.-P. Remenieras, and F. Patat, "Transit time ultrasonic flowmeter: velocity profile estimation," in Proc. IEEE Ultrasonics Symposium, september 2005.

[2] G. Wendt, B. Mickan, R. Kramer, and D. Dopheide, "Systematic investigation of pipe flows and installation effects using laser Doppler anemometry - part I. profile measurement downstream of several pipe configurations and flow conditioners." Flow Measurement and Instrumentation, vol. 7, no. 3/4, pp. 141-149, 1996.

[3] L. Salami, "On velocity-area methods for asymetric profiles," University of Southampton interim Report V, 1972.

[4] R. Gordon, "A tutorial on art," IEEE Trans. on Nuclear Science, vol. 21, pp. 78-93, 1974. 